\title{
De novo genome assembly of Cercospora beticola for microsatellite marker development and validation
}

\author{
Niloofar Vaghefi a , Julie R. Kikkert ${ }^{\text {b}}$, Melvin D. Bolton ${ }^{\text {c, e }}$, Linda E. Hanson ${ }^{\text {d }}$, Gary A.
}

Secor ${ }^{\mathrm{e}}$, Sarah J. Pethybridge ${ }^{\mathrm{a}, \text { * }}$

${ }^{a}$ School of Integrative Plant Sciences, Plant Pathology \& Plant-Microbe Biology Section, Cornell University, Geneva, NY, USA

${ }^{\mathrm{b}}$ Cornell Cooperative Extension, Canandaigua, NY, USA

${ }^{c}$ USDA - ARS, Northern Crop Science Laboratory, Fargo, ND, USA

${ }^{\mathrm{d}}$ USDA - ARS, Sugar Beet and Bean Research Unit, Michigan State University, MI, USA

${ }^{\mathrm{e}}$ Department of Plant Pathology, North Dakota State University, Fargo, ND, USA

* Corresponding author. E-mail address: sjp277@ cornell.edu (S. J. Pethybridge). 


\begin{abstract}
Cercospora leaf spot caused by Cercospora beticola is a significant threat to the production of sugar and table beet worldwide. A de novo genome assembly of $C$. beticola was used to develop eight polymorphic and reproducible microsatellite markers for population genetic analyses. These markers were used, along with five previously described microsatellite loci to genotype two $C$. beticola populations from table beet fields in New York, USA. High allelic and genotypic diversity and low population differentiation was found between fields. Linkage disequilibrium of loci after clone-correction of datasets was attributed to the presence of two distinct clonal lineages within the populations. Linkage equilibrium of loci in one of the clusters supported the presence of sexual reproduction. The draft de novo genome assembly will help elucidate the reproductive system of $C$. beticola through investigating evidence of recombination in the $C$. beticola genome.
\end{abstract}

Keywords: Beta vulgaris, microsatellite, population genetics, SSR, sugar beet, swiss chard, table beet 


\section{Introduction}

Cercospora beticola is a hemibiotrophic ascomycete that causes Cercospora leaf spot (CLS) on Beta vulgaris worldwide. On sugar beet (B. vulgaris subsp. vulgaris), significant reductions in root weight and extractable sucrose, together with costs of disease management, result in significant annual losses from CLS (Franc, 2010). On table beet (B. vulgaris subsp. vulgaris), losses are predominantly associated with deterioration of foliage, which interferes with mechanical harvesting that relies upon leaves to pull the root from the ground. Moreover, CLS is a major constraint to table beet and swiss chard (B. vulgaris subsp. cicla) production for the fresh market as disease symptoms render the leaves unmarketable.

The taxonomic position of $C$. beticola was recently resolved based on a five-locus phylogeny (Groenewald et al., 2005). This analysis separated C. beticola from C. apii, a morphologically indistinguishable species causing CLS on celery (Apium graveolens) (Groenewald et al., 2005). Cercospora apii is phylogenetically closely-related to $C$. beticola, and has also been reported on sugar beet but its low prevalence is considered inconsequential to CLS epidemics in the genus Beta (Groenewald et al., 2005, 2006).

A sexual form for $C$. beticola has not been identified but it is believed to have the potential for sexual reproduction to occur in a heterothallic manner based on the presence of the two alternate mating type alleles (MAT1-1-1 and MAT1-2-1) in equal distribution in some sugar beet and table beet fields in Europe and US (Bolton et al., 2012b; Groenewald et al., 2006, 2007; Vaghefi et al., 2016). Population genetic studies have also provided indirect evidence for potential cryptic sex of $C$. beticola based on linkage equilibrium of genetic markers and high genotypic diversity of $C$. beticola populations (Groenewald et al., 2007, 2008; Vaghefi et al., 2016) while no evidence for sexual reproduction was detected in some parts of the US and also the middle east (Bakhshi et al., 2011; Obuya et al., 2011). In the absence of a known sexual form, C. beticola is believed to mainly overwinter on plant debris as pseudostromata (Franc, 2010), which may survive in soil for a maximum of 22 months to three years, depending on the climatic conditions (Khan et al., 2008; Nagel, 1938; Solel, 1970).

Various molecular markers have been used to characterize C. beticola populations, including random amplified polymorphic DNA (RAPD) (Moretti et al., 2004; Weiland et al., 2001), direct amplification of minisatellite region DNA (DAMD) (Moretti et al., 2004, 2006), and amplified 
fragment length polymorphism (AFLP) (Groenewald et al., 2008; Turgay et al., 2010; Weiland et al., 2001). Groenewald et al. (2007) used a combination of three single nucleotide polymorphism markers (SNP), one restriction fragment length polymorphism (RFLP) and five microsatellites for genetic characterization of $C$. beticola populations from sugar beet in Europe, Iran and New Zealand; and detected high genotypic diversity and linkage equilibrium of loci, indicative of random mating in $C$. beticola populations. The five microsatellite loci developed by Groenewald et al. (2007) were used to genotype two small $C$. beticola populations $(n=24)$ from table beet fields, confirming high genotypic diversity and linkage equilibrium of loci following clone correction, also interpreted as potential for sexual reproduction of $C$. beticola in New York, USA (Vaghefi et al., 2016).

Microsatellites are widely used for population genetic studies due to their co-dominance, hyper-variability, locus-specificity, reproducibility and relative abundance in the genome of all organisms (Bruford and Wayne, 1993). Although some of the properties that make microsatellites desirable may also confound population genetic inferences (e.g., high mutation rates resulting in high rates of homoplasy and ascertainment bias), with the appropriate choice of computational techniques and integration of multiple analyses to answer relevant questions, microsatellites can be very powerful population genetic tools. Microsatellites are especially suitable for studying recent phylogeographic and demographic events within a species or among closely-related species (Banke and McDonald, 2005; Linde, 2010; Putman and Carbone, 2014). Only five microsatellite markers are currently available for C. beticola (Groenewald et al., 2007), which lack the power to capture the entire genotypic diversity present in $C$. beticola populations in table beet fields in New York (unpublished data). Thus, additional polymorphic microsatellite markers are required to enable more robust statistical quantification of the genetic diversity in $C$. beticola populations.

Development of microsatellite markers was historically arduous requiring the construction of small-insert genomic libraries, Southern hybridization for detecting positive clones, plasmid isolation and sequencing, which was later facilitated through employing microsatellite-enriched genomic libraries (Zane et al., 2002). Following the advent of massively parallel sequencing approaches, microsatellite discovery has become faster and cheaper. Despite the utility of raw sequence reads for microsatellite discovery (Castoe et al. 2010; O'Bryhim et al., 2012), short 
reads limit the identification of microsatellites due to the lack of sufficient flanking regions for primer design, and also sequence redundancy, making the design of locus-specific primers challenging (Castoe et al. 2010). Alternatively, partial assembly of short but high quality Illumina reads has become the superior method for the identification of microsatellites (Cai et al., 2013; Mercière et al., 2015; Muller et al., 2016; Vaghefi et al., 2015). Despite its global importance, no genome sequence data is currently available for $C$. beticola.

The objectives were to (i) produce a de novo genome assembly of $C$. beticola to identify a set of polymorphic and reproducible microsatellite markers; (ii) validate the utility of the developed markers for genetic analyses of $C$. beticola populations; and (iii) test the transferability of the markers to the phylogenetically-close species, C. apii.

\section{Materials and methods}

\subsection{Fungal material, isolation and DNA extraction}

A total of 118 C. beticola isolates were included in this study. Isolate Tb14-085, collected from a table beet field in New York, USA, was used to generate a de novo genome assembly for microsatellite library development. The identity of this isolate was confirmed as C. beticola based on the phylogenetic analysis of the concatenated internal transcribed spacer (ITS) region of the nrDNA, along with actin, calmodulin, histone $\mathrm{H} 3$ and translation elongation factor 1- $\alpha$ sequences (data not shown). Thirty-six C. beticola isolates collected from swiss chard, sugar beet and table beet in various geographical locations were used to screen the markers for polymorphism (Table S1). This included the ex-epitype strain of C. beticola, CBS 116456, obtained from the Centraalbureau voor Schimmelcultures (CBS), Utrecht, the Netherlands. The ex-epitype strain of $C$. apii, CBS 116455, was used to test the transferability of the developed markers to $C$. apii. Eighty-three $C$. beticola isolates collected from two processing table beet fields ( $n=39$ and 44 from Field 1 and Field 2, respectively) in upstate New York, in 2014, were included to assess the utility of the microsatellite loci for capturing the genotypic diversity of $C$. beticola populations. A subset of these isolates $(n=48)$ were previously characterized using the five microsatellite loci developed by Groenewald et al. (2007) in a prior study (Vaghefi et al. 2016). The procedures used for isolations from plant material and DNA extraction from these isolates were as described in Vaghefi et al. (2016). For the remainder of the samples, isolations were conducted according to Bolton et al. (2012a). For DNA extraction, single-conidium isolates 
were grown in clarified V8 broth ((10 \% (v/v) clarified V8 juice (Campbell's Soup Co., USA), $\left.0.5 \%(\mathrm{w} / \mathrm{v}) \mathrm{CaCO}_{3}\right)$ for four to seven days before mycelia were harvested and lyophilized. For each isolate, 20 to $25 \mathrm{mg}$ of the lyophilized mycelia was ground to a fine powder in a $2 \mathrm{ml}$ microcentrifuge tube using two 4.5-mm diameter, zinc-plated, spherical balls (Daisy premium grade BBs, Rogers, AR) in a tissue homogenizer (TissueLyser, Qiagen, Valencia, CA). Genomic DNA for each isolate was extracted using a DNA extraction kit (DNeasy Plant Minikit, Qiagen) according to the manufacturer's instructions. Quantity of the extracted DNA was determined using a fluorometer (Qubit 2.0, Invitrogen, Inc., Grand Island, NY). The integrity of the DNA samples was checked through electrophoresis on a $1 \%$ agarose gel $(1 \% \mathrm{wt} / \mathrm{vol}$ agarose in Trisacetate-EDTA) amended with $0.5 \times$ (v/v) nucleic acid stain GelRed (Biotium, Inc., Hayward, CA).

\subsection{De novo genome assembly}

A total of 5.6 $\mu \mathrm{g}$ genomic DNA of isolate Tb14-085 was used to prepare a PCR-free library with average insert of $\sim 550 \mathrm{bp}$, at the Cornell University Institute of Biotechnology Genomics Facility, Ithaca, New York, USA. The PCR-free library was made using Illumina's TruSeq Nano DNA LT Sample Preparation kits, using the manufacturer's protocol. The library was sequenced using the Illumina paired-end ( $2 \times 300$ bp) MiSeq platform. This yielded 4,607,564

paired reads totaling $\sim 2.7 \mathrm{~Gb}$. Quality control of the produced sequences was done using FastQC v.0.11.2 (http://www.bioinformatics.bbsrc.ac.uk/projects/fastqc) in the GALAXY portal (Afgan et al., 2016).

The Kmer counting software Jellyfish v.2.2.3 (Marçais and Kingsford, 2011) was used to estimate the genome size using the raw Illumina sequences. Frequencies of 51-, 61-, 71- and 81mers occurring in paired-end reads were counted, and the read sequencing depth $(\mathrm{N})$ was estimated according to the formula; $\mathrm{M}=\mathrm{N} \times(\mathrm{L}-\mathrm{K}+1) / \mathrm{L}$, where $\mathrm{M}$ is the peak frequency of each k-mer, $\mathrm{L}$ is the read length, and $\mathrm{K}$ is the k-mer size. Genome size was approximated by dividing the total sequence length by the read sequencing depth. Genome coverage was then estimated by dividing the total sequence data by the estimated genome size.

Two approaches were taken to produce a partial de novo assembly of the C. beticola genome. First, the raw sequence data was assembled in DISCOVAR de novo v.52488, which is a small genome assembler designed for de novo assembly of long Illumina paired-end sequences from a 
single PCR-free library (Weisenfeld et al., 2014). A second de novo assembly was generated using SOAPdenovo2 v.1.05 ( $\mathrm{Li}$ et al., 2008). For this, the raw sequences were first qualitytrimmed using Trimmomatic v.0.36 (Bolger et al., 2014), with a 4-base long sliding window, a minimum quality value of 28 , and minimum length of 80 bases. Of the total of 4,607,564 input read pairs, 3,488,133 paired reads $(75.70 \%)$ and 752,300 single reads $(16.33 \%)$ were retained for assembly after quality trimming (7.97\% discarded). SOAPdenovo-127mer was used to conduct partial genome assemblies testing the kmer length parameter between 71 and 127, in 2 bases increments, as well as mix kmer parameters. The completeness of the final genome assemblies was assessed using BUSCO v.1.2 (Simão et al., 2015), which employs sets of Benchmarking Universal Single-Copy Orthologs (BUSCO) for Fungi (Waterhouse et al., 2013) to measure genome completeness.

\subsection{Microsatellite marker development}

The assembly obtained from DISCOVAR de novo resulted in the highest N50 value and was hence retained for microsatellite library development. The standard scaffold fasta sequences (flattened lines) obtained by the highest coverage paths through the assembled edges were screened for microsatellite motifs using the software Tandem Repeat Finder v.408 (Benson, 1999). The search was conducted for all the tri- to hexa-nucleotide repeat motifs with a minimum score of 50 and a matching point of two. Microsatellite loci were chosen based on the contig size and the number of repeat motifs. In order to minimize the probability of the physical linkage of loci, only one microsatellite locus in each scaffold was chosen for primer design. To increase the

probability of polymorphism, loci with at least seven repeat units were included. The scaffolds containing the five microsatellite loci designed by Groenewald et al. (2007) were excluded. Also, to ensure neutrality of the loci, sequences containing the microsatellite motifs were blasted against the non-redundant database of the NCBI to ascertain they did not belong to genic regions. Twenty-four loci were selected for primer design using the program Primer3 v.0.4.0 (Untergasser et al., 2012). All parameters were set as default, except for a more stringent range of melting temperature $\left(59^{\circ} \mathrm{C}<\mathrm{Tm}<61{ }^{\circ} \mathrm{C}\right.$ ), with product sizes ranging from 100 to $420 \mathrm{bp}$, to maximize the potential for multiplexing.

The designed primers were tested for amplification against six C. beticola isolates (CBS 116456, Ch15-029; HI-Ch-131, Tb14-085, Tb14-259, and Tb15-306; Table S1). The PCR mix 
for each primer pair $(15 \mu$ l total volume) contained $1 \times$ Standard PCR buffer (New England Biolabs, Inc., Ipswich, MA), $1.5 \mathrm{mM} \mathrm{MgCl}_{2}$ (New England Biolabs), $0.1 \mathrm{mM}$ dNTPs (New England Biolabs), $0.2 \mu \mathrm{M}$ of each primer, $1 \mathrm{U}$ Taq polymerase (New England Biolabs), and 10 ng of template DNA. The PCR conditions included an initial denaturation for 5 min at $95{ }^{\circ} \mathrm{C}$; followed by 36 cycles of denaturation at $95{ }^{\circ} \mathrm{C}$ for $30 \mathrm{~s}$, annealing at 55 and $59{ }^{\circ} \mathrm{C}$ for $20 \mathrm{~s}$, and extension at $68{ }^{\circ} \mathrm{C}$ for $30 \mathrm{~s}$; and a final extension at $68{ }^{\circ} \mathrm{C}$ for $5 \mathrm{~min}$. A final set of 10 primer pairs, which resulted in single bands of the expected size in all DNA samples tested, were chosen for subsequent tests for polymorphism. To confirm the presence of microsatellite repeats, PCR amplicons were sequenced at the Cornell University Institute of Biotechnology Genomic Diversity Facility using on an ABI 3730xl sequencer.

\subsection{Microsatellite screening for polymorphism and multiplexing}

For the 10 selected primer pairs, the 5' end of the forward primer was labeled with one of the four fluorophores (6-FAM, NED, PET and VIC; Applied Biosystems, Foster City, CA) to enable multiplexing. PCRs were performed using the genomic DNA of 36 geographically diverse isolates (Table S1), as described above, at an annealing temperature of $55{ }^{\circ} \mathrm{C}$. Fluorescentlabeled products were resolved on an ABI 3730xl DNA Analyzer at the Cornell University Institute of Biotechnology Genomic Diversity Facility, using a GeneScan-500 LIZ size standard (Applied Biosystems). PCRs and fragment analyses were replicated across all 36 isolates to ensure reproducibility of allele size calls. Alleles were scored using the Geneious Microsatellite Plugin Version 1.4 (Geneious Version 7.1.8, http://www.geneious.com; Kearse et al. 2012). A locus was considered polymorphic if it resulted in at least two alleles, and the most frequent allele had a maximum frequency of 0.99. As a result, eight loci were identified as polymorphic. Loci summary statistics were calculated in GenAlEx v.6.502 (Peakall and Smouse, 2006, 2012) (Table 1).

The eight polymorphic markers were divided into two multiplex PCR sets, each containing four primer pairs (Table 2) using Multiplex Manager v.1.2 (Holleley and Geerts, 2009). The minimum distance between the loci was set to $50 \mathrm{bp}$ and a complementary threshold of seven was allowed. Multiplex reactions were performed in $15 \mu$ volumes consisting of $10 \mathrm{ng}$ template DNA, $3 \mu \mathrm{L}$ of the Multiplex PCR 5× Master Mix (New England BioLabs Inc.), and the 
corresponding fluorescent-labeled primers at variable concentrations from 0.1 to $0.3 \mu \mathrm{M}$ used to optimize the primer concentration for each locus.

\subsection{Cross-species amplification}

The eight markers developed here and five microsatellite loci reported by Groenewald et al. (2007) were tested for amplification against the ex-epitype strain of $C$. apii, CBS 116455 . The PCR mix for each primer pair $(15 \mu$ l total volume) contained $1 \times$ Standard PCR buffer (New England Biolabs, Inc., Ipswich, MA), $1.5 \mathrm{mM} \mathrm{MgCl}_{2}$ (New England Biolabs), $0.1 \mathrm{mM}$ dNTPs (New England Biolabs), $0.15 \mu \mathrm{M}$ of each primer, $1 \mathrm{U}$ Taq polymerase (New England Biolabs), and $10 \mathrm{ng}$ of template DNA. The PCR conditions included an initial denaturation for $5 \mathrm{~min}$ at 95 ${ }^{\circ} \mathrm{C}$; followed by 38 cycles of denaturation at $95{ }^{\circ} \mathrm{C}$ for $30 \mathrm{~s}$, annealing at $58{ }^{\circ} \mathrm{C}$ for $30 \mathrm{~s}$, and extension at $68{ }^{\circ} \mathrm{C}$ for $30 \mathrm{~s}$; and a final extension at $68{ }^{\circ} \mathrm{C}$ for $5 \mathrm{~min}$. The PCRs were replicated three times to ensure reproducibility. Fragment analysis of the loci that consistently amplified a single band in C. apii was conducted as above.

\subsection{Genotyping of Cercospora beticola populations from table beet in New York}

Eighty-three $C$. beticola isolates, collected from two processing table beet fields in New York in 2014 (44 isolates from Field 1; 39 isolates from Field 2), situated $300 \mathrm{~m}$ apart from each other, were genotyped using 13 microsatellite loci. The eight polymorphic markers developed here were amplified in two multiplex reactions, and five microsatellite loci developed by Groenewald et al. (2007) were amplified in a third multiplex mix at optimized primer concentrations (Table 2). For capillary electrophoresis, multiplex mixes 1 and 2 were pooled together into one bin (Table 2). Fragment analysis and allele calling were performed as described. PCRs and fragment analyses were replicated for $50 \%$ of the isolates, including all isolates with null or rare alleles, to reduce genotyping error and confirm reproducibility.

\subsection{Data analysis}

Nei's (1978) measure of allelic diversity, allelic richness and number of private alleles were estimated in GenAlEx. Number of multi-locus genotypes (MLGs), expected number of MLGs after rarefaction (eMLG), and evenness ( $\left.\mathrm{E}_{5}\right)$ were estimated using poppr v.2.2.0 (Kamvar et al., 2014, 2015). Genotypic diversity ( $\lambda$ ) was also calculated in poppr, and was corrected for sample size by multiplying by N/(N-1). Lambda $(\lambda)$ was originally described by Simpson (1949) as the 
probability that two individuals chosen at random and independently from the population belong to the same genotype ( $\sum_{i=1}^{R} P_{i}^{2}$, where $P_{i}$ is the frequency of the ith genotype and $\mathrm{R}$ is the total number of genotypes). However, the measure of genotypic diversity calculated in poppr as $\lambda$ is described as one minus the sum of squared genotype frequencies (http://grunwaldlab.github.io/Population_Genetics_in_R/Genotypic_EvenRichDiv.html), i.e., the probability that two randomly selected genotypes are different (Simpson's complement index), and ranges from zero (only one genotype exists in the population) to one (all genotypes are different). In order to investigate the ability of the microsatellite loci to capture the genotypic diversity of the $C$. beticola populations, the plot of mean genotypic diversity against the number of loci was obtained using MultiLocus v.1.3 (Agapow and Burt, 2001).

As a measure of population differentiation, pairwise $\Phi_{\mathrm{PT}}$ (Excoffier et al., 1992) which is analogous to $\mathrm{F}_{\mathrm{ST}}$ and based on genetic distance estimates that assume a stepwise mutation model, was calculated in GenAlEx v.6.502 (Peakall and Smouse, 2006, 2012) and its statistical significance was tested through 999 randomizations. Jost's (2008) measure of population differentiation (D) was estimated using the package mmod in R (Winter, 2012). In contrast to $\mathrm{F}_{\mathrm{ST}}$ and its derivatives, D estimates population differentiation based on the effective number of alleles rather than heterozygosity. Confidence intervals (95\%) for D were estimated after 1,000 bootstrap simulations. Principal Coordinate Analysis (PCoA) of genetic distances among MLGs was conducted in the R package adegenet v.2.0.1 (Jombart, 2008).

To investigate the underlying structure without a priori assumption of populations, three approaches were used. The number of underlying clusters were inferred in the Bayesian clustering software STRUCTURE v.2.3.4 (Pritchard et al., 2000) using a burn-in period of 100,000 Monte Carlo Markov Chain, 1,000,000 iterations for data collection, and 10 simulations for each $K$ ( $K=1$ to 10$)$. The optimal value of $K$ was chosen by computing $\Delta K$ (Evanno et al., 2005) using STRUCTURE HARVETSER v.0.6.94 (Earl and vonHoldt, 2012). The 10 replicated runs for the optimal $\mathrm{K}$ were combined using CLUMMP v.1.1.2 (Jakobsson and Rosenberg, 2007) to generate a single output which was visualized using DISTRUCT v.1.1 (Rosenberg, 2004). Discriminant Analysis of Principal Components (DAPC) was conducted using adegenet, which is independent of Hardy-Weinberg equilibrium and linkage equilibrium of loci assumptions. The optimal number of clusters were determined using the function find.clusters, 
retaining all principal components. DAPC was then used to assign individuals into clusters, retaining the number of principal components encompassing $96 \%$ of the cumulative variance. As a third measure of investigating population structure, a distance-based dendogram using Bruvo's distance (Bruvo et al., 2004), was plotted using poppr with 1,000 bootstrap replicates. Bootstrap branch support values greater than $50 \%$ were retained. All analyses of structure were conducted on both the clone-corrected and non-clone-corrected data sets.

To investigate the null hypothesis of random mating, and thus random association of loci, the standardized index of association $\left(\overline{r_{d}}\right)$ (Agapow and Burt 2001), scaled for the number of loci, was estimated after 1,000 permutations in poppr, for both clone-corrected and non-clone corrected datasets from each field. Apart from clonal reproduction, linkage of loci could also be caused by population structure/admixture (Milgroom, 1996). Therefore, the standardized index of association was also estimated for the two clusters detected by STRUCTURE, separately, before and after clone-correction. Smaller population sizes after clone-correction may result in loss of power for detection of linkage disequilibrium (LD) (Brown, 1975). We adopted the method by Liu et al. (1996) to ensure that small sample sizes did not inhibit detection of LD by drawing small random sub-samples from each cluster 20 times and estimating the index of association and its significance by 999 bootstrap permutations.

\section{Results}

\subsection{De novo genome assembly}

The Illumina paired-end ( $2 \times 300 \mathrm{bp})$ MiSeq sequencing of C. beticola isolate Tb14-085 resulted in a total of 9,215,128 reads with mean base quality of 28.5. The estimated genome size of $C$. beticola using variable k-mer lengths in Jellyfish was $\sim 37.7 \mathrm{Mb}$, based on which a genome coverage of $\sim 74 \times$ was approximated. Both DISCOVAR de novo and SOAPdenovo generated assemblies of high quality with $97 \%$ completeness based on their content of Benchmarking Universal Single-Copy Orthologs (Table 3). However, DISCOVAR de novo out-performed SOAPdenovo by producing significantly larger scaffolds with higher N50 values. The highest N50 value using SOAPdenovo was obtained when a mixed kmer of 71 and 77 was used, resulting in a scaffold $\mathrm{N} 50$ of $597.83 \mathrm{Kbp}$ and a total assembly size of $35.88 \mathrm{Mbp}$. The draft genome assembly obtained using DISCOVAR de novo had a total assembly size of $35.36 \mathrm{Mbp}$, with a scaffold N50 value of $1.02 \mathrm{Mbp}$ (Table 3). 


\subsection{Microsatellite library development and genotyping}

Of the ten microsatellites tested against 36 C. beticola isolates, eight loci were polymorphic. Sequencing of these loci confirmed the presence of the target repeat motifs (GenBank accession nos $\underline{\mathbf{K X 4 5 2 3 5 0}}$ to $\underline{\mathbf{K X 4 5 2 3 5 7}}$; Table 1). Allelic diversity ranged from 0.32 to 0.67 , and the number of alleles per locus ranged from three to eight. Replicating the PCRs and fragment analyses for these microsatellite loci confirmed reproducibility of the allele size calls. The eight polymorphic loci were successfully amplified in two four-plex PCRs and subsequently pooled into one bin for capillary separation (Table 2).

Following genotyping of $83 \mathrm{C}$. beticola isolates using 13 microsatellite loci (Table 2), locus SSRCb4 was discarded from the final data set because it consistently amplified two distinct peaks of 158 and $190 \mathrm{bp}$ in $6 \%$ of the isolates. This was not attributed to DNA contamination as the remaining loci resulted in a single peak in all isolates. Further BLAST searches of the SSRCb4 sequence ( $\underline{\mathbf{D Q 9 0 2 5 6 7}}$ ) against the assembled genome of $C$. beticola (isolate Tb14-085) detected multiple fragments with high similarity to SSRCb4 occurring in different scaffolds. A sequence with 97\% similarity to SSRCb4 (excluding the differences in the repeat region) was identified in scaffold 205, and multiple other sequences with 86 to $91 \%$ similarity to SSRCb4 in scaffolds $8,10,18,28,40,62,105,134$ and 145. All these fragments included primer binding sites with 55 to $100 \%$ similarity to the sequence of SSRCb4F and SSRCb4R, which could have resulted in the amplification of the additional band.

\subsection{Cross-species transferability}

Of the 13 microsatellite loci tested, only SSRCb3, SSRCb21, SSRCb22, SSRCb24, SSRCb25, SSRCb26 and SSRCb27 consistently amplified a single band in C. apii strain CBS 116455. Cercospora apii shared common alleles with $C$. beticola at all of these loci except for SSRCb27 (Table 2).

\subsection{Genotyping of Cercospora beticola populations from table beet in New York}

Cercospora beticola populations from table beet fields showed high levels of gene diversity (Field $1=0.45$ and Field $2=0.43$ ) and genotypic diversity (Field $1=0.975$ and Field $2=0.979$ ) (Table 4). In the total of 39 and 44 C. beticola isolates from Field 1 and Field 2, respectively, 28 and 33 MLGs were detected, indicating < 30\% clonality in each field (Table 4). Of the 15 
recurrent MLGs (occurring more than once), four MLGs were shared between the two fields. Genotypic diversity was found to plateau as a function of the number of loci indicating that sufficient microsatellite markers had been used to capture the genetic diversity of the populations (Fig. 1).

Very low but significant genetic differentiation was detected between the $C$. beticola populations from the two fields with only $5.6 \%$ of the total variation attributed between fields $\left(\Phi_{\mathrm{PT}}=0.056, P=0.008\right)$, which did not decrease much following clone-correction $\left(\Phi_{\mathrm{PT}}=0.049\right.$, $P=0.030)$. Jost's measure of differentiation also depicted low level of genetic differentiation based on allele frequencies $(\mathrm{D}=0.078,95 \% \mathrm{CI}: 0.056-0.103)$. This was corroborated by the PCoA results that showed overlap of MLGs from the two fields and lack of population differentiation between fields (Fig. 2). However, there was clustering of isolates along the principal coordinates.

Bayesian cluster analysis of both the clone-corrected and non-clone corrected datasets within STRUCTURE indicated the presence of two distinct clusters in the population (Fig. 3). This was congruent with the dendrogram produced based on Bruvo's distance, in which $C$. beticola isolates were grouped into two major clades (Fig. S1). Sixty-three isolates were assigned to cluster 1 and 20 isolates were assigned to cluster 2 (posterior probability of memberships > $0.92)$. These two clusters were significantly genetically differentiated $\left(\Phi_{\mathrm{PT}}=0.507, P=0.001\right)$, did not correspond to sampling location. The two groups also showed significant difference in allelic diversity as only $33 \%$ of the loci were polymorphic in cluster 2 (Table 5).

DAPC analysis of the non-clone-corrected data set resulted in an inconclusive value for $\mathrm{K}$ as the Bayesian Information Criterion (BIC) consistently decreased with the number of clusters until $\mathrm{K}$ reached the total number of MLGs $(\mathrm{K}=57)$. Therefore, the optimal $\mathrm{K}$ was determined as the number of clusters where BIC decreased by a negligible amount, i.e., $\mathrm{K}=4$ (Fig. 4). On the other hand, DAPC analysis of the clone-corrected dataset resulted in the lowest BIC value for K $=6$ (Fig. S2). Twenty isolates that were assigned to cluster 2 using STRUCTURE remained assigned to a distinct cluster in the DAPC analyses (group 2 in Fig. 4b; group 3 in Fig. S1), and the 63 isolates assigned to cluster 1 by STRUCTURE were further divided into distinct genetic groups in the DAPC analyses. 
The standardized index of association $\left(\overline{r_{d}}\right)$ detected significant non-random association of alleles in both fields in the clone-corrected and non-clone-corrected datasets for populations from each field, which rejected the null hypothesis of random mating. When index of association was estimated for each of the clusters detected by STRUCTURE (Fig. 3), significant linkage of loci was detected within cluster $1(P=0.001)$ and cluster $2(P=0.018)$. However, following clone correction of the datasets, the individuals in both clusters were in linkage equilibrium $(P>$ 0.056). Clone-correction resulted in smaller population sizes in both clusters ( $n=45$ and 12 in clusters 1 and 2, respectively). To ensure that failure to detect LD in the clone-corrected datasets was not an artefact of small sample sizes, index of association was estimated for random subsamples of 45 individuals from cluster 1, and 12 individuals from cluster 2. In all sub-samples drawn from cluster 1, linkage disequilibrium was detected, which indicated that 45 samples were sufficient to detect linkage of loci. For the random sub-samples from cluster 2, the index of association failed to detect $\mathrm{LD}$ in $55 \%$ of the simulations, indicating that 12 samples were too small to provide sufficient power to detect LD.

\section{Discussion}

A de novo genome assembly of $C$. beticola was used to develop eight highly polymorphic and reproducible microsatellite markers, six of which were transferable to $C$. apii. These markers, combined with four loci developed by Groenewald et al. (2007) were sufficient in depicting the genotypic diversity present in two $C$. beticola populations and thus provide a valuable tool for population genetic studies. The developed markers were only tested against the ex-type isolate of C. apii, thus, their polymorphism and suitability for population genetic studies of $C$. apii requires further investigation. Cercospora apii was first described from celery in Germany, and is suspected to have originated in Western Europe (Groenewald et al., 2005). It has been hypothesized that $C$. apii was originally able to compete with $C$. beticola and infect sugar beet in the early 1900s, when C. apii strains isolated from sugar beet were submitted to the CBS-KNAW fungal collection. However, isolations from sugar beet fields in 2000s failed to isolate C. apii (Groenewald et al., 2005). Considering the suitability of microsatellite loci for studying recent phylogeographic events among closely-related species (Putman and Carbone, 2014), markers developed here may be used to test hypotheses regarding the speciation of C. apii and C. beticola in future studies. 
The de novo assembly constructed in DISCOVAR de novo using 300 bp paired-end reads from a PCR-free library of a single insert size resulted in a high quality assembly with a contig N50 value of $\sim 461 \mathrm{Kbp}$ and a scaffold N50 value larger than $1 \mathrm{Mb}$. The assembly produced using SOAPdenovo resulted in lower N50 values but had a similar level of completeness (97\%) based on content of Benchmarking Universal Single-Copy Orthologs. Cercospora beticola was estimated to have a genome size of $\sim 37 \mathrm{Mb}$, which is smaller than the assembled genomes of $C$. arachidicola (46.5 Mb; Orner et al., 2015), C. zeina (45.2 Mb; Zaccaron et al., 2015), and C. zeae-maydis (46.6 Mb; http://genome.jgi.doe.gov/Cerzm1/Cerzm1.info.html), and larger than $C$. kikuchii (28.4 Mb; Higtower et al., 1995), C. sojina (31.1 Mb) and C. cf. flagellaris (33.2 Mb) (Zaccaron et al., 2015).

Quantification of the population structure in the $C$. beticola isolates detected variable numbers of clusters which could not be attributed to sampling locations. Two major clusters of individuals were detected in STRUCTURE, with no admixture between the clusters as each individual was distinctly assigned to only one cluster with posterior probabilities of $>0.92$ (Fig. 3b). These two clusters showed significant genetic differentiation $\left(\Phi_{\mathrm{PT}}=0.507\right)$, which was higher than the genetic differentiation between the populations from the two fields $\left(\Phi_{\mathrm{PT}}=0.056\right)$. These two major clonal lineages did not correspond to sampling location and occurred sympatrically in both fields. A five-locus phylogeny of a subset of isolates (ITS, actin, calmodulin, histone 3 and translation elongation factor 1- $\alpha$; data not shown) revealed that the two clusters detected here did not correspond to the two $C$. beticola clades detected based on the sequence of histone $\mathrm{H} 3$ region in a previous study (Groenewald et al., 2013).

In DAPC analysis of the entire data set using the function find.clusters in adegenet, no stabilization of BIC values were detected. However, $\mathrm{K}=4$ was selected to best explain diversity present in the $C$. beticola population since the BIC value decreased only incrementally when $\mathrm{K}>$ 4 (Fig. 4a). Absence of individuals belonging to more than one cluster indicated lack of admixture among these clusters (Fig. 4c). Due to an increase in $\Delta \mathrm{K}$ at $\mathrm{K}=4$ in the STRUCTURE analysis (Fig. 3), we also determined the clusters assigned by STRUCTURE at K = 4 (data not shown), which did not correspond to the four groups detected through DAPC analysis. The BIC graph for the clone-corrected data set showed a clear decrease of BIC until $\mathrm{K}=6$, after which BIC started to increase, indicating six clusters best explained the data (Fig. S2). In either case, 
the detected clusters could not be attributed to sampling locations/fields. DAPC analysis is a powerful technique for population structure inference, and may be superior to STRUCTURE in inferring number of subpopulations (Jombart et al. 2010). However, inferring the number of clusters using the BIC criterion is not always explicitly clear and the number of clusters retained is an arbitrary choice often guided by biological significance of the detected clusters (Jombart and Collins, 2015). Use of clustering algorithms for microsatellite data has other drawbacks, e.g., lack of information regarding their performance and cluster validation methods (Putman and Carbone, 2014). Thus, multiple approaches should be incorporated to draw conclusions from microsatellite data sets.

As a third measure of investigating population structure, a distance-based dendogram using Bruvo's distance (Bruvo et al., 2004), was also created (Fig. S2). The two clusters detected in STRUCTURE, corresponded to the two major clusters in the dendrogram (Fig. S2). However, most of the clusters assigned by the DAPC analyses did not correspond to a distinct cluster in the dendrogram detected by Bruvo's distance (Fig. S2), which may be indicative of admixture in $C$. beticola population.

No sexual form has been identified for $C$. beticola but the presence of cryptic sex has been suggested based on the equal frequencies of the mating type alleles, high genotypic diversity and linkage equilibrium of microsatellite loci in some $C$. beticola populations (Groenewald et al., 2007; Bolton et al., 2012b). High genotypic diversity detected in the $C$. beticola populations from table beet fields in New York is consistent with presence of sexual recombination. However, significant linkage disequilibrium, even after clone-correction, is expected from asexually reproducing populations (Milgroom, 1996). In a previous study, genotyping of a subset of isolates $(n=48)$ collected from the same fields using five microsatellite markers did not detect linkage disequilibrium of the loci after clone correction of the datasets (Vaghefi et al., 2016). Larger sample sizes used in this study enhanced the power of the LD analysis. The availability of additional microsatellite loci also contributed to a higher discriminative power to distinguish between genotypes, resulting in larger population sizes after clone-correction. This emphasizes that relatively large samples sizes are required when testing for linkage disequilibrium of genetic markers (Lenski, 1993; Milgroom, 1996). 
Apart from clonal reproduction, linkage disequilibrium of loci may be caused by other factors such as physical linkage of loci, population structure/admixture and random genetic drift (Milgroom, 2015). In order to minimize the probability of the physical linkage of loci, we designed only one microsatellite locus in each scaffold. SSRCb2 and SSCb3 (Groenewald et al., 2007) were later identified to be present on the same scaffold but $\sim 42,000$ bp apart from each other. Eliminating SSRCb2 and SSRCb3 from the datasets and re-calculation of the index of association provided similar results (data not shown), discounting that the LD detected in this study could be attributed to linkage of these loci.

Since strong clustering of isolates into two non-admixed groups was detected (Fig. 3), the LD analysis was repeated for each of these clusters. The null hypothesis of random mating was not rejected for the isolates in cluster 1 , which indicated admixture within this cluster. The LD analysis for cluster 2, however, was inconclusive as the sample size was prohibitively small. Multiple private alleles at seven microsatellite loci were the major contributors to genetic differentiation of these two clonal lineages. The biological significance of this population structure needs further investigations. Interestingly, all the 20 isolates in cluster 2 belonged to one mating-type (MAT-1-1-1; data not shown), which might indicate lack of sexual recombination within this cluster. Further monitoring of the private alleles within the C. beticola populations in subsequent years, and detection of their probable flow between the two clusters may provide evidence of recombination in C. beticola populations in New York.

The de novo assembly of $C$. beticola enabled development of highly polymorphic and reproducible microsatellite loci, which, in combination with previously developed markers (Groenewald et al., 2007), result in greater statistical power for population genetic studies on $C$. beticola. Moreover, availability of a draft genome of $C$. beticola will enable more advanced genotyping techniques such as Genotyping by Sequencing (GBS) (Elshire et al., 2011), which will provide thousands of SNPs to test for the signatures of recombination, or investigate presence of signs of sex, e.g., meiosis related genes, in the genome of C. beticola (Milgroom et al., 2014).

\section{Acknowledgments}


This research was supported by the United States Department of Agriculture, National Institute of Food and Agriculture Hatch project NYG-625424, managed by the New York Agricultural Experiment Station (NYSAES), Cornell University, Geneva, NY, USA, and the NYSAES Director's Controlled Endowment Fund. Thanks to Ms. Traci Hoogland and Mr. David Strickland for excellent technical support, and table beet growers for allowing access to fields for sample collection. Mention of trade names or commercial products in this article is solely for the purpose of providing specific information and does not imply recommendation or endorsement by the U.S. Department of Agriculture. We also thank the anonymous reviewers for constructive suggestions on an earlier version on this manuscript. 


\section{References}

Agapow, P.M., Burt, A., 2001. Indices of multilocus linkage disequilibrium. Mol. Ecol. Notes 1, $101-102$.

Afgan, E., Baker, D., van den Beek, M., Blankenberg, D., Bouvier, D., Čech, M., Chilton, J., Clements, D., Coraor, N., Eberhard, C., Grüning, B., 2016. The Galaxy platform for accessible, reproducible and collaborative biomedical analyses: 2016 update. Nucleic Acids Res. kw343.

Banke, S., McDonald, B.A., 2005. Migration patterns among global populations of the pathogenic fungus Mycosphaerella graminicola. Mol. Ecol. 14, 1881-1896.

Benson, G., 1999. Tandem repeats finder: A program to analyze DNA sequences. Nucleic Acids Res. 27, 573-580.

Bolger, A.M., Lohse, M., Usadel, B., 2014. Trimmomatic: a flexible trimmer for Illumina sequence data. Bioinformatics btu170.

Bakhshi, M., Arzanlou, M., Babai-Ahari, A., 2011. Uneven distribution of mating type alleles in Iranian populations of Cercospora beticola, the causal agent of Cercospora leaf spot disease of sugar beet. Phytopathol. Mediterr. 50, 101-109.

Bolton, M.D., Birla, K., Rivera, V., Rudolph, K.D, Secor, G.A., 2012a. Characterization of CbCyp51 from field isolates of Cercospora beticola Phytopathology 102, 298-305.

Bolton, M.D., Secor, G.A., Rivera, V., Weiland, J.J., Rudolph, K., Birla, K., Rengifo, J., Campbell, L.G., 2012b. Evaluation of the potential for sexual reproduction in field populations of Cercospora beticola from USA. Fungal Biol. 116, 511-521.

Brown, A.H.D., 1975. Sample sizes required to detect linkage disequilibrium between two or three loci. Theor. Popul. Biol. 8, 184-201.

Bruford, M.W., Wayne, R.K., 1993. Microsatellites and their application to population genetic studies. Curr. Opin. Genet. Dev. 3, 939-943.

Bruvo, R., Michiels, N. K., D’Souza, T. G., Schulenburg, H., 2004. A simple method for the calculation of microsatellite genotype distances irrespective of ploidy level. Mol. Ecol. 13, 21012106. 
Cai, G., Leadbetter, C.W., Muehlbauer, M.F., Molnar, T.J., Hillman, B.I., 2013. Genome-Wide microsatellite identification in the fungus Anisogramma anomala using Illumina sequencing and genome assembly. PLoS ONE 8, e82408.

Castoe, T. A., Poole, A. W., Gu, W., Jason de Koning, A. P., Daza, J. M., Smith, E. N., Pollock, D. D., 2010. Rapid identification of thousands of copperhead snake (Agkistrodon contortrix) microsatellite loci from modest amounts of 454 shotgun genome sequence. Mol. Ecol. Res. $10: 341-347$.

Earl, D. A., vonHoldt, B. M., 2012. STRUCTURE HARVESTER: a website and program for visualizing STRUCTURE output and implementing the Evanno method. Conserv. Genet. Resour. 4,359-361.

Elshire, R.J., Glaubitz, J.C., Sun, Q., Poland, J.A., Kawamoto, K., Buckler, E.S., Mitchell, S.E., 2011. A robust, simple genotyping-by-sequencing (GBS) approach for high diversity species. PloS one 6, e19379.

Evanno, G., Regnaut, S., Goudet, J., 2005. Detecting the number of clusters of individuals using the software STRUCTURE: a simulation study. Mol. Ecol. 14, 2611-2620.

Excoffier, L., Smouse, P.E., Quattro, J.M., 1992. Analysis of molecular variance inferred from metric distances among DNA haplotypes: application to human mitochondrial DNA restriction data. Genetics 131, 479-491.

Franc, G.D., 2010. Ecology and epidemiology of Cercospora beticola, in: Lartey, R.T., Weiland, J.J., Panella, L., Crous, P.W., Windels, C.E. (Eds.), Cercospora Leaf Spot of Sugar Beet and Related Species. American Phytopathological Society, St. Paul, MN, pp. 7-19.

Groenewald, M., Groenewald, J.Z., Braun, U., Crous, P.W., 2005. Distinct species exist within the Cercospora apii morphotype. Phytopathology 95, 951-959.

Groenewald, M., Groenewald, J.Z., Harrington, T.C., Abeln, E.C., Crous, P.W., 2006. Mating type gene analysis in apparently asexual Cercospora species is suggestive of cryptic sex. Fung. Genet. Biol. 43, 813-825. 
Groenewald, M., Groenewald, J.Z., Linde, C.C., Crous, P.W., 2007. Development of polymorphic microsatellite and single nucleotide polymorphism markers for Cercospora beticola (Mycosphaerellaceae). Mol. Ecol. Notes 7, 890-892.

Groenewald, M., Linde, C.C., Groenewald, J.Z., Crous, P.W., 2008. Indirect evidence for sexual reproduction in Cercospora beticola populations from sugar beet. Plant Pathol. 57, 25-32.

Groenewald, J.Z., Nakashima, C., Nishikawa, J., Shin, H.D., Park, J.H., Jama, A.N., Groenewald, M., Braun, U., Crous, P.W., 2013. Species concepts in Cercospora: spotting the weeds among the roses. Stud. Mycol. 75, 115-170.

Higtower, R.C., Callahan, M., Upchurch, R.G., 1995. Electrophoretic karyotype of Cercospora kikuchii. Curr. Genet. 27, 290-292.

Holleley, C.E., Geerts, P.G., 2009. Multiplex Manager 1.0: a cross-platform computer program that plans and optimizes multiplex PCR. Biotechniques 46, 511-517.

Jakobsson, M., Rosenberg, N.A., 2007. CLUMPP: a cluster matching and permutation program for dealing with label switching and multimodality in analysis of population structure.

Bioinformatics 23, 1801-1806.

Jombart, T., 2008. adegenet: a $\mathrm{R}$ package for the multivariate analysis of genetic markers. Bioinformatics 24, 1403-1405.

Jombart, T., Collins, C., 2015. A tutorial for discriminant analysis of principal components (DAPC) using adegenet 2.0. 0. http://adegenet.r-forge.r-project.org/files/tutorial-dapc.pdf.

Jombart, T., Devillard, S., Balloux, F., 2010. Discriminant analysis of principal components: a new method for the analysis of genetically structured populations. BMC Genet. 11, 1.

Jost, L., 2008. GST and its relatives do not measure differentiation. Mol. Ecol. 17, 4015-4026.

Kamvar, Z.N., Tabima, J.F., Grünwald, N.J., 2014. Poppr: an R package for genetic analysis of populations with clonal, partially clonal, and/or sexual reproduction. PeerJ 2, e281.

Kamvar, Z.N., Brooks, J.C., Grünwald, N.J., 2015. Novel R tools for analysis of genome-wide population genetic data with emphasis on clonality. Front. Genet. 6, 208. 
Kearse, M., Moir, R., Wilson, A., Stones-Havas, S., Cheung, M., Sturrock, S., Buxton, S., Cooper, A., Markowitz, S., Duran, C., Thierer, T., Ashton, B., Mentjies, P., Drummond, A., 2012. Geneious Basic: an integrated and extendable desktop software platform for the organization and analysis of sequence data. Bioinformatics 28, 1647-1649.

Khan, J., del Rio, L.E., Nelson, R., Rivera-Varas, V., Secor, G.A., Khan M.F.R., 2008, Survival, dispersal, and primary infection site for Cercospora beticola in sugar beet. Plant Dis. 92, 741745.

Lenski, R.E., 1993. Assessing the genetic structure of microbial populations. Proc. Natl. Acad. Sci. 90, 4334-4336.

Li, I., Li, Y., Kristiansen, K., Wang, J., 2008. SOAP: short oligonucleotide alignment program. Bioinformatics 24, 713-714.

Linde, C., 2010. Population genetic analyses of plant pathogens: new challenges and opportunities. Austral. Plant Pathol. 39, 23-28.

Liu, Y.C., Cortesi, P., Double, M.L., MacDonald, W.L. Milgroom, M.G., 1996. Diversity and multilocus genetic structure in populations of Cryphonectria parasitica. Phytopathology 86, 1344-1351.

Ludwig, J.A., Reynolds, J.F., 1988. Statistical Ecology: A Primer on Methods and Computing. John Wiley \& Sons, New York.

Marçais, G., Kingsford, C., 2011. A fast, lock-free approach for efficient parallel counting of occurrences of k-mers. Bioinformatics 27, 764-770.

Mercière, M., Laybats, A., Carasco-Lacombe, C., Tan, J.S., Klopp, C., Durand-Gasselin, T., Alwee, S.S.R.S., Camus-Kulandaivelu, L., Breton, F., 2015. Identification and development of new polymorphic microsatellite markers using genome assembly for Ganoderma boninense, causal agent of oil palm basal stem rot disease. Mycol. Prog. 14, 1-11.

Milgroom, M.G., 1996. Recombination and the multilocus structure of fungal populations. Annu. Rev. Phytopathol. 34, 457-477. 
Milgroom, M.G., 2015. Population Biology of Plant Pathogens: Genetics, Ecology, and Evolution. APS Press, St. Paul, Minnesota, USA.

Milgroom, M.G., Jiménez-Gasco, M., García, M.O., Drott, M.T, Jiménez-Díaz, R.M., 2014. Recombination between clonal lineages of the asexual fungus Verticillium dahliae detected by genotyping by sequencing. PloS one 9, e106740.

Moretti, M., Karaoglanidis, G., Saracchi, M., Fontana, A., Farina, G., 2006. Analysis of genotypic diversity in Cercospora beticola Sacc. field isolates. Ann. Microbiol. 56, 215-221.

Moretti, M., Saracchi, M., Farina, G., 2004. Morphological, physiological and genetic diversity within a small population of Cercospora beticola Sacc. Ann. Microbiol. 54, 129-150.

Muller, M.F., Barnes, I., Kunene, N.T., Crampton, B.G., Bluhm, B.H., Phillips, S., Olivier N.A., Berger, D.K, 2016. Cercospora zeina from maize in South Africa exhibits high genetic diversity and lack of 2 regional population differentiation. Phytopathology First Look doi: 10.1094/PHYTO-02-16-0084-FI.

Nagel, C.M., 1938. The longevity of Cercospora beticola in soil. Phytopathology 28, 342-350.

Nei, M., 1987. Molecular Evolutionary Genetics. Columbia University Press, NY.

O’Bryhim, J., Chong, J P., Lance, S.L., Jones, K.L., Roe, K.J., 2012. Development and characterization of sixteen microsatellite markers for the federally endangered species: Leptodea leptodon (Bivalvia: Unionidae) using paired-end Illumina shotgun sequencing. Conserv. Genet. Resour. 4, 787-789.

Obuya, J.O., Hanson, L.E., Franc, G.D., 2011. Mating type idiomorphs distribution and their correlation to benzimidazole-resistance in Cercospora beticola from the Central High Plains region, USA. American Society of Sugar Beet Technologists, Proceedings from the 36th Biennial Meeting doi: pp.10.5274.ASSBT.2011.114.

Orner, V.A., Cantonwine, E.G., Wang, X.M., Abouelleil, A., Bochicchio, J., Nusbaum, et al., 2015. Draft genome sequence of Cercospora arachidicola, causal agent of early leaf spot in peanuts. Genome Announc. 3, e01281-15. 
Peakall, R., Smouse, P.E., 2012. GenAlEx 6.5: genetic analysis in Excel. Population genetic software for teaching and research - an update. Bioinformatics 28, 2537-2539.

Peakall, R., Smouse, P.E., 2006. GENALEX 6: genetic analysis in Excel. Population genetic software for teaching and research. Mol. Ecol. Notes. 6, 288-295.

Pritchard, J.K., Stephens, M., Donnelly, P., 2000. Inference of population structure using multilocus genotype data. Genetics 155, 945-959.

Putman, A.I., Carbone, I., 2014. Challenges in analysis and interpretation of microsatellite data for population genetic studies. Ecol. Evol. 4, 4399-4428.

Rosenberg, N.A., 2004. DISTRUCT: a program for the graphical display of population structure. Mol. Ecol. Notes 4, 137-138.

Simão, F.A., Waterhouse, R.M., Ioannidis, P., Kriventseva, E.V., Zdobnov, E.M., 2015. BUSCO: assessing genome assembly and annotation completeness with single-copy orthologs. Bioinformatics 31, 3210-3212.

Simpson, E.H., 1949. Measurement of diversity. Nature 163, 688.

Solel, Z., 1970. Survival of Cercospora beticola, the causal agent of sugar beet leaf spot, in Israel. Trans. Br. Mycol. Soc. 54, 504-506.

Turgay, E.B., Bakir, M., Ozeren, P., Katircioglu, Y.Z., Maden, S., 2010. Detection of pathotypes and genetic diversity of Cercospora beticola. Plant Pathol. J. 26, 306-312.

Untergasser, A., Cutcutache, I., Koressaar, T., Ye, J., Faircloth, B.C., Remm, M., Rozen, S.G., 2012. Primer3 - new capabilities and interfaces. Nucleic Acids Res. 40, e115

Vaghefi, N., Hay, F.S., Ades, P.K., Pethybridge, S.J., Ford, R. and Taylor, P.W., 2015. Rapid changes in the genetic composition of Stagonosporopsis tanaceti population in Australian pyrethrum fields. Phytopathology 105, 358-369.

Vaghefi, N., Hay, F.S., Kikkert, J.R., Pethybridge, S.J., 2016. Genotypic diversity and resistance to azoxystrobin of Cercospora beticola on processing table beet in New York. Plant Dis. 100, 1466-1473. 
Waterhouse, R.M., Tegenfeldt, F., Li, J., Zdobnov, E.M., Kriventseva, E.V., 2013. OrthoDB: a hierarchical catalog of animal, fungal and bacterial orthologs. Nucleic Acids Res. 41, D358D365.

Weiland, J., Eide, J., Rivera, V.V., Secor, G., 2001. Genetic diversity of Cercospora beticola in the US and association of molecular markers with tolerance to the fungicide triphentylin hydroxide (TPTH). (Abstr.) Phytopathology 91, S94.

Weisenfeld, N.I., Yin, S., Sharpe, T., Lau, B., Hegarty, R., Holmes, L., Sogoloff, B., Tabbaa, D., Williams, L., Russ, C., Nusbaum, C., 2014. Comprehensive variation discovery in single human genomes. Nat. Genet. 46, 1350-1355.

Winter, D.J., 2012. MMOD: an R library for the calculation of population differentiation statistics. Mol. Ecol. Res. 12, 1158-1160.

Zaccaron, A., Ridenour, J, Smith, J., Sharma, S., Lawson, N., Zaccaron, M.L., Fakhoury, A., Bluhm, B., 2015. Molecular Mechanisms of Cercospora Pathogenicity Revealed Through Comparative Genomics. Plant and Animal Genome XXIII Conference. Plant and Animal Genome, 2015.

Zane, L., Bargelloni, L., Patarnello, T., 2002. Strategies for microsatellite isolation: a review. Mol. Ecol. 11, 1-16. 
Table 1 Characteristics of the eight polymorphic microsatellite markers developed for Cercospora beticola.

\begin{tabular}{|c|c|c|c|c|c|c|c|}
\hline SSR locus & Scaffold $^{\text {a }}$ & $\begin{array}{l}\text { Scaffold } \\
\text { length } \\
\text { (bp) }\end{array}$ & $\begin{array}{l}\text { Repeat } \\
\text { motif }^{b}\end{array}$ & Forward primer ( $5^{\prime}$ to $\left.3^{\prime}\right)$ & $\mathrm{N}_{\mathrm{a}}^{\mathrm{c}}$ & $\mathrm{H}_{\mathrm{e}}^{\mathrm{d}}$ & $\begin{array}{l}\text { GenBank } \\
\text { accession } \\
\text { no. }\end{array}$ \\
\hline SSRCb20 & 137 & 42,968 & (TAGA)7 & $\begin{array}{l}\text { F: AGCGCGCTTAGGTAATCTTTCTA } \\
\text { R: AAACTAAATAGGCTATTAGGACTTTAGGAA }\end{array}$ & 6 & 0.47 & KX452350 \\
\hline $\mathrm{SSRCb} 21$ & 2 & $1,940,376$ & $(\mathrm{AGC}) 12$ & $\begin{array}{l}\text { F: GACTTTGGCATTCGAGAAGATGG } \\
\text { R: CCACTAAACGTATCTCTTTGCTGT }\end{array}$ & 8 & 0.67 & KX452351 \\
\hline $\mathrm{SSRCb} 22$ & 94 & 371,411 & $(\mathrm{GAA}) 9$ & $\begin{array}{l}\text { F: GCCACTTCATTACCACCTTGAAT } \\
\text { R: TGAGCTGATGTGAAAGGTAGAGG }\end{array}$ & 4 & 0.34 & KX452352 \\
\hline $\mathrm{SSRCb} 23$ & 80 & 221,449 & $($ GCGGAG)6 & $\begin{array}{l}\text { F: CGGTGAATGTTGTTGGTAGGTTT } \\
\text { R: TGGTCGGTCTTTCTCACATGAAC }\end{array}$ & 5 & 0.32 & KX452353 \\
\hline $\mathrm{SSRCb} 24$ & 10 & $1,240,088$ & (TGT)13 & $\begin{array}{l}\text { F: GTGGAATAAGAGGATGAGGGGAC } \\
\text { R: TCTCAATCTCAGCTCTCACACAC }\end{array}$ & 4 & 0.58 & KX452354 \\
\hline $\mathrm{SSRCb} 25$ & 46 & 497,910 & $(\mathrm{GAG}) 11$ & $\begin{array}{l}\text { F: GACGAGCATTCCATTGAGAAGTC } \\
\text { R: TCGTCGTTTTGGTCCTCTTCTTC }\end{array}$ & 6 & 0.64 & KX452355 \\
\hline SSRCb26 & 6 & $1,321,140$ & $(\mathrm{ACACG}) 7$ & $\begin{array}{l}\text { F: GTGGAAGAGGGTGAAGAGGTG } \\
\text { R: CAGTAGGCGGGGAGTTGTG }\end{array}$ & 3 & 0.42 & KX452356 \\
\hline $\mathrm{SSRCb} 27$ & 0 & $3,283,681$ & $(\mathrm{CAA}) 24$ & $\begin{array}{l}\text { F: CGTCAAAGCAGTCCCTCGAT } \\
\text { R: AATTGAACAAGCGCCCAACC }\end{array}$ & 7 & 0.50 & KX452357 \\
\hline
\end{tabular}

${ }^{\mathrm{a}}$ Location of the loci in the draft genome sequence of $C$. beticola isolate Tb14-085 obtained from DISCOVAR de novo.

${ }^{\mathrm{b}}$ Number of repeats are based on the sequence of $C$. beticola isolate Tb14-085.

${ }^{\mathrm{c}}$ Number of alleles.

${ }^{\mathrm{d}}$ Nei's allelic diversity (Nei, 1987). 
Table 2 Attributes of a microsatellite library used for genotyping Cercospora beticola populations collected from processing table beet fields in New York, USA in 2014; and transferability to C. apii.

\begin{tabular}{|c|c|c|c|c|c|}
\hline SSR locus $^{\mathrm{a}}$ & $\begin{array}{l}\text { Fluorescent } \\
\text { dye }\end{array}$ & Allele sizes (bp) in C. beticola & $\begin{array}{l}\text { Primer concentration } \\
(\mu \mathrm{M})\end{array}$ & $\begin{array}{l}\text { Multiplex } \\
\operatorname{mix}\end{array}$ & $\begin{array}{l}\text { Allele sizes in } C . \text { apii }^{\text {b }} \\
\text { strain CBS } 116455\end{array}$ \\
\hline SSRCb20 & 6-FAM & $156,160,164,168,172,180$ & 0.15 & 1 & na \\
\hline $\mathrm{SSRCb} 21$ & VIC & $165,168,174,177,180,186,189,192$ & 0.18 & 2 & $165 \mathrm{bp}$ \\
\hline $\mathrm{SSRCb} 22$ & NED & $185,188,191,194$ & 0.15 & 1 & $194 \mathrm{bp}$ \\
\hline $\mathrm{SSRCb} 23$ & NED & $269,281,287,299,305$ & 0.12 & 2 & na \\
\hline $\mathrm{SSRCb} 24$ & VIC & $313,316,319,331$ & 0.12 & 2 & $331 \mathrm{bp}$ \\
\hline $\mathrm{SSRCb} 25$ & PET & $234,252,255,258,261,264$ & 0.225 & 1 & $234 \mathrm{bp}$ \\
\hline $\mathrm{SSRCb} 26$ & VIC & $354,359,364$ & 0.225 & 1 & $354 \mathrm{bp}$ \\
\hline $\mathrm{SSRCb} 27$ & 6-FAM & $368,374,398,428,431,434,482$ & 0.12 & 2 & $404 \mathrm{bp}$ \\
\hline SSRCb1 & NED & $219,221,223$ & 0.12 & 3 & na \\
\hline $\mathrm{SSRCb} 2$ & 6-FAM & 195,197 & 0.12 & 3 & na \\
\hline SSRCb3 & 6-FAM & $\begin{array}{l}258,260,266,272,274,290,294,298,300, \\
310,312,314,316,318,320,322\end{array}$ & 0.3 & 3 & $260 \mathrm{bp}$ \\
\hline $\mathrm{SSRCb} 4$ & PET & $158,174,190$ & 0.3 & 3 & na \\
\hline SSRCb6 & VIC & $225,227,229,231,233,241,243,245$ & 0.12 & 3 & na \\
\hline
\end{tabular}

SSRCb1, SSRCb2, SSRCb3, SSRCb4 and SSRCb6 were developed by Groenewald et al. (2007); SSRCb20 to 27 were developed in this study.

${ }^{\mathrm{b}}$ na $=$ did not amplify or resulted in poor and non-reproducible amplification. 
Table 3 Statistics for the Cercospora beticola de novo assemblies produced from Illumina paired-end MiSeq sequencing of strain Tb14-085.

\begin{tabular}{|c|c|c|c|c|c|c|c|c|}
\hline $\begin{array}{l}\text { Assembly } \\
\text { software }\end{array}$ & Genome completeness $^{\mathrm{a}}$ & $\begin{array}{l}\text { Assembly } \\
\text { size }(\mathrm{Mb})\end{array}$ & $\begin{array}{l}\text { No. of } \\
\text { scaffolds }\end{array}$ & $\begin{array}{l}\text { Longest } \\
\text { scaffold } \\
\text { (bp) }\end{array}$ & $\begin{array}{l}\text { Scaffold } \\
\mathrm{N} 50^{\mathrm{b}}(\mathrm{bp})\end{array}$ & $\begin{array}{l}\text { Scaffold } \\
\text { N90 }(\mathrm{bp})\end{array}$ & $\begin{array}{l}\text { No. of } \\
\text { contigs }\end{array}$ & $\begin{array}{l}\text { Contig } \\
\text { N50 } \\
\text { (bp) }\end{array}$ \\
\hline $\begin{array}{l}\text { DISCOVAR } \\
\text { de novo }\end{array}$ & $\begin{array}{l}\text { 97\% (C: 1401, F: 30, M: 7, T: } \\
1438)\end{array}$ & 35.4 & 291 & $3,283,681$ & $1,023,488$ & 200,981 & 699 & 461,091 \\
\hline SOAPdenovo & $\begin{array}{l}97 \% \text { (C: } 1400, \mathrm{~F}: 30, \mathrm{M}: 8, \mathrm{~T}: \\
1438)\end{array}$ & 35.9 & 400 & $2,579,648$ & 597,830 & 95,645 & 8,323 & 249,547 \\
\hline
\end{tabular}

${ }^{\mathrm{a}}$ Summary completeness assessment in BUSCO notation; $\mathrm{C}=$ complete single-copy BUSCOs, $\mathrm{F}=$ fragmented BUSCOs; $\mathrm{M}=$ missing BUSCOs, $\mathrm{T}=$ total BUSCO groups searched.

${ }^{\mathrm{b}}$ length of the shortest scaffold among those that collectively cover $50 \%$ of the assembly.

${ }^{c}$ length of the shortest scaffold among those that collectively cover $90 \%$ of the assembly 
Table 4 Genetic diversity indices for Cercospora beticola populations collected from two processing table beet fields in New York, USA, 2014.

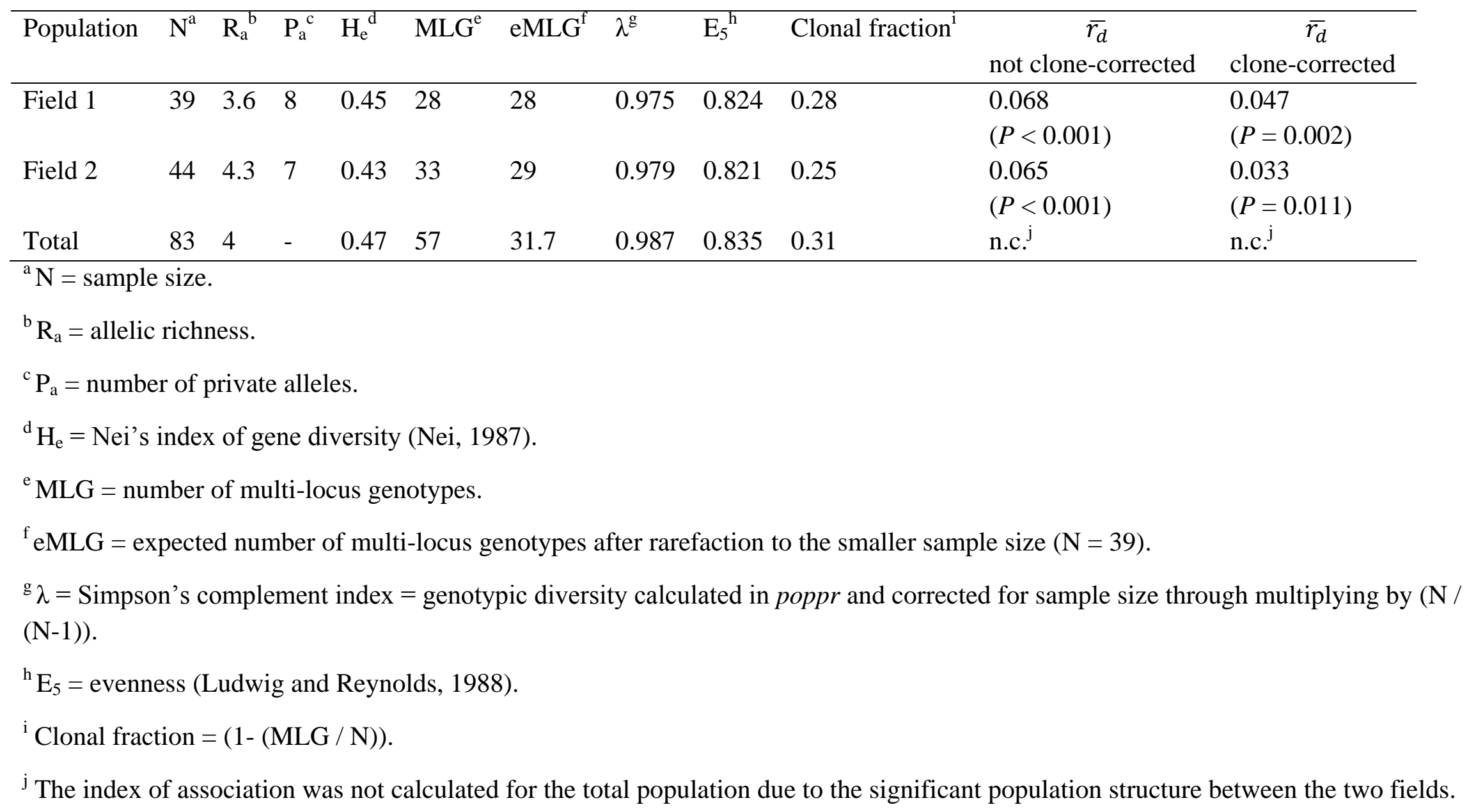


Table 5 Allelic diversity of the two clonal lineages detected with the software, STRUCTURE, of Cercospora beticola isolated from two processing table beet fields in New York, USA.

\begin{tabular}{|c|c|c|c|c|c|c|}
\hline \multirow[t]{2}{*}{ locus } & \multicolumn{3}{|c|}{ Cluster $1(n=63)$} & \multicolumn{3}{|c|}{ Cluster $2(n=20)$} \\
\hline & $\mathrm{N}_{\mathrm{a}}^{\mathrm{a}}$ & $\mathrm{P}_{\mathrm{a}}^{\mathrm{b}}$ & $\mathrm{H}_{\mathrm{e}}^{\mathrm{c}}$ & $\mathrm{N}_{\mathrm{a}}^{\mathrm{a}}$ & $\mathrm{P}_{\mathrm{a}}^{\mathrm{b}}$ & $\mathrm{H}_{\mathrm{e}}{ }^{\mathrm{c}}$ \\
\hline SSRCb1 & 2 & 1 & 0.20 & 1 & 0 & 0.00 \\
\hline $\mathrm{SSRCb} 2$ & 2 & 1 & 0.23 & 1 & 0 & 0.00 \\
\hline SSRCb3 & 15 & 11 & 0.91 & 5 & 1 & 0.75 \\
\hline SSRCb6 & 7 & 5 & 0.84 & 2 & 0 & 0.52 \\
\hline SSRCb20 & 3 & 2 & 0.40 & 1 & 0 & 0.00 \\
\hline SSRCb21 & 4 & 3 & 0.64 & 1 & 0 & 0.00 \\
\hline SSRCb22 & 4 & 3 & 0.21 & 1 & 0 & 0.00 \\
\hline $\mathrm{SSRCb} 23$ & 3 & 2 & 0.20 & 1 & 0 & 0.00 \\
\hline $\mathrm{SSRCb} 24$ & 3 & 3 & 0.32 & 1 & 1 & 0.00 \\
\hline $\mathrm{SSRCb} 25$ & 5 & 4 & 0.63 & 1 & 0 & 0.00 \\
\hline SSRCb26 & 3 & 1 & 0.40 & 2 & 0 & 0.40 \\
\hline SSRCb27 & 3 & 2 & 0.23 & 2 & 1 & 0.52 \\
\hline Average over all loci & 4.50 & - & 0.43 & 1.58 & - & 0.18 \\
\hline
\end{tabular}

${ }^{\mathrm{a}}$ Number of alleles.

${ }^{\mathrm{b}}$ Number of private alleles.

${ }^{\mathrm{c}}$ Nei's allelic diversity (Nei, 1987). 
Fig. 1. Mean genotypic diversity as a function of the number of loci for 12 microsatellite loci used for population genetic analysis of Cercospora beticola isolates collected from two processing table beet fields in New York. The horizontal axis represents the number of loci randomly sampled. The vertical axis shows the observed genotypic diversity.

Fig. 2. Principal coordinate analysis (PCoA) of Cercospora beticola isolates from two processing table beet fields in New York. Red and green clusters represent populations from fields 1 and 2, respectively.

Fig. 3. Detection of two clusters in the Cercsopora beticola population collected from two processing table beet fields in New York using the software, STRUCTURE. A, estimation of $\Delta \mathrm{K}$ to determine the optimal K (number of clusters) based on likelihood method (Evanno et al., 2005). B, assignment of $C$. beticola isolates to clusters based on 10 replicated runs at $\mathrm{K}=2$. The two clusters are distinguished by the colors, blue and orange. Each bar represents one isolate and the bar height represents estimated membership fraction of each individual in each of the inferred clusters. Clusters 1 and 2 included 63 and 20 isolates, respectively. The clusters did not correspond to sampling location and occurred sympatrically in both fields.

Fig. 4. Discriminant Analysis of Principal Components (DAPC) for Cercospora beticola isolates from two processing table beet fields in New York. A, Bayesian Information Criterion (BIC) plotted against the number of inferred clusters for the non-clone-corrected data set. B, ordination plot of 4 assigned genetically related groups of individuals. Group 2 corresponds to the 20 isolates identified as cluster 2 in STRUCTURE. The remaining three groups were detected as a single cluster (cluster 1) in STRUCTURE. C, membership probability of $C$. beticola isolates to the four genetic clusters detected through DAPC analysis. Each bar indicates an individual and the colors represent their membership in the predicted clusters. 


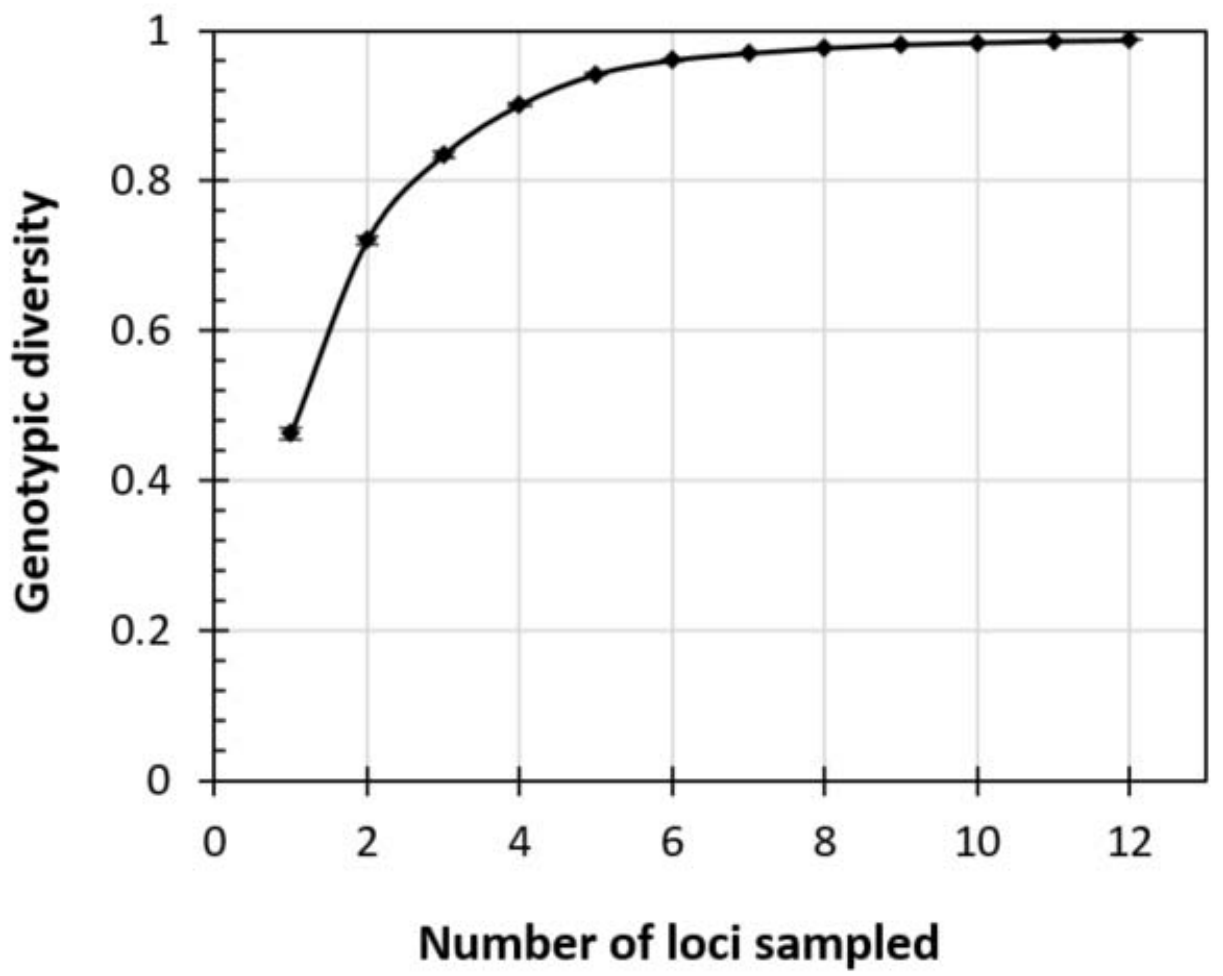




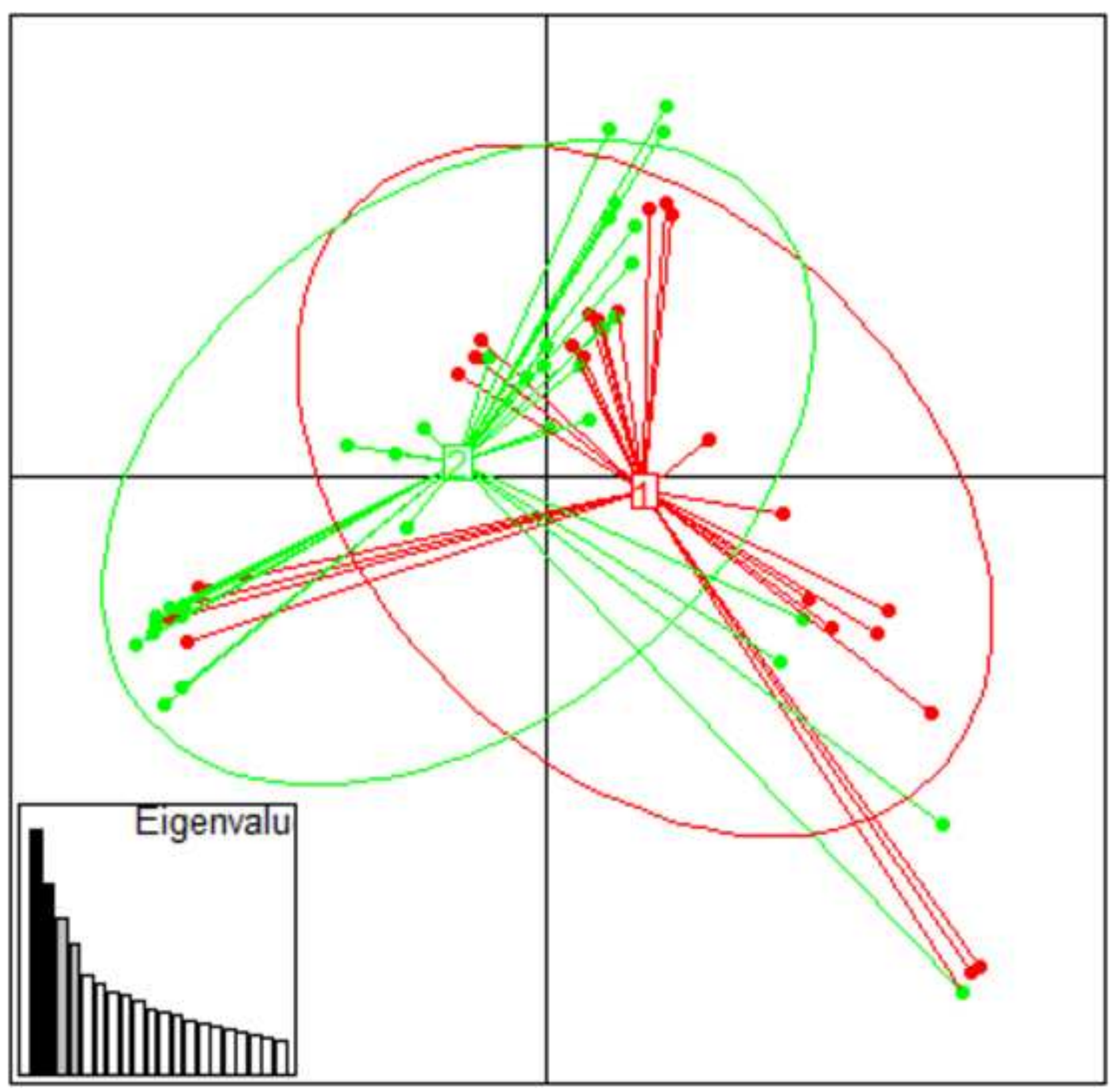



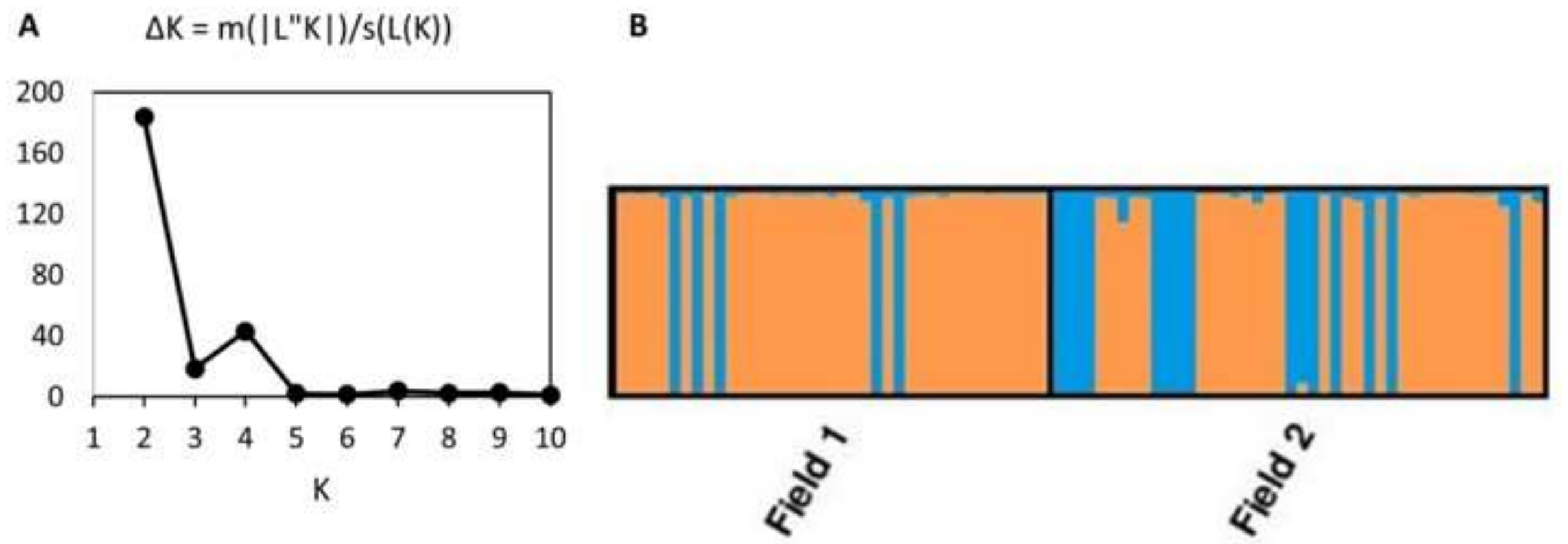

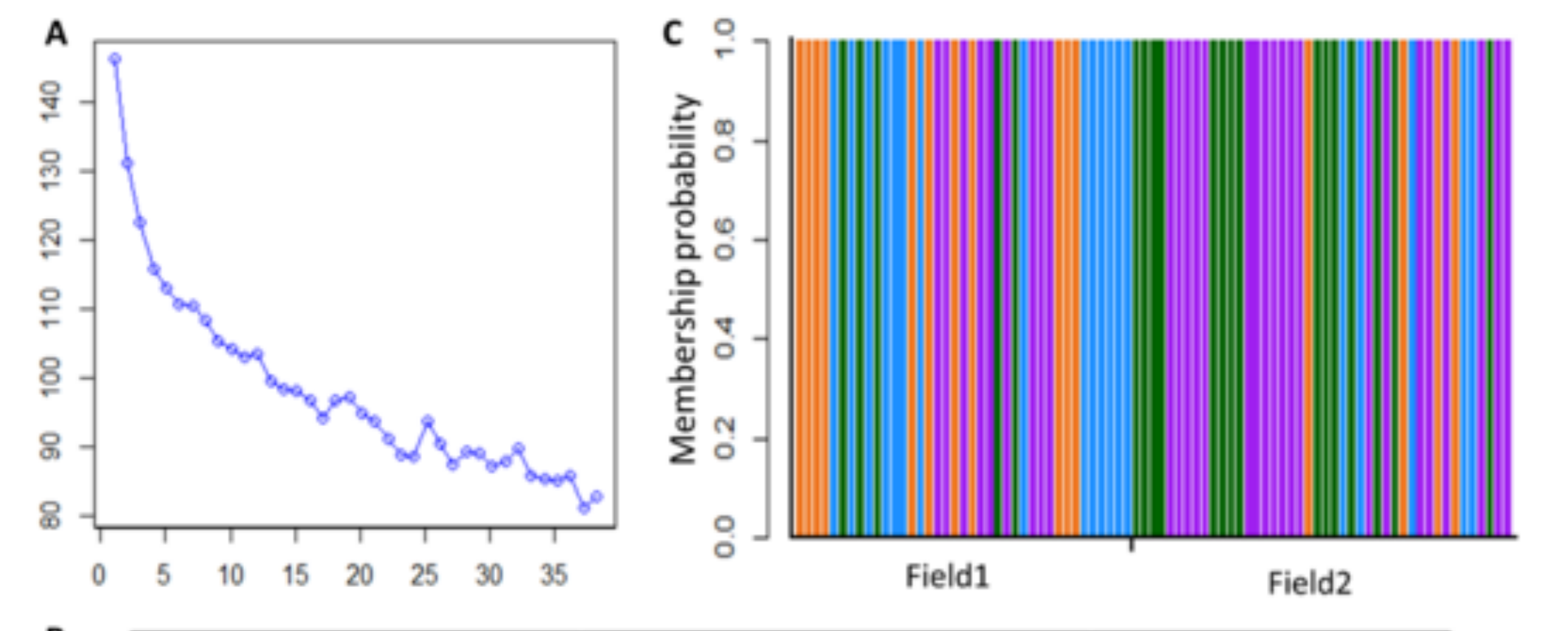

B

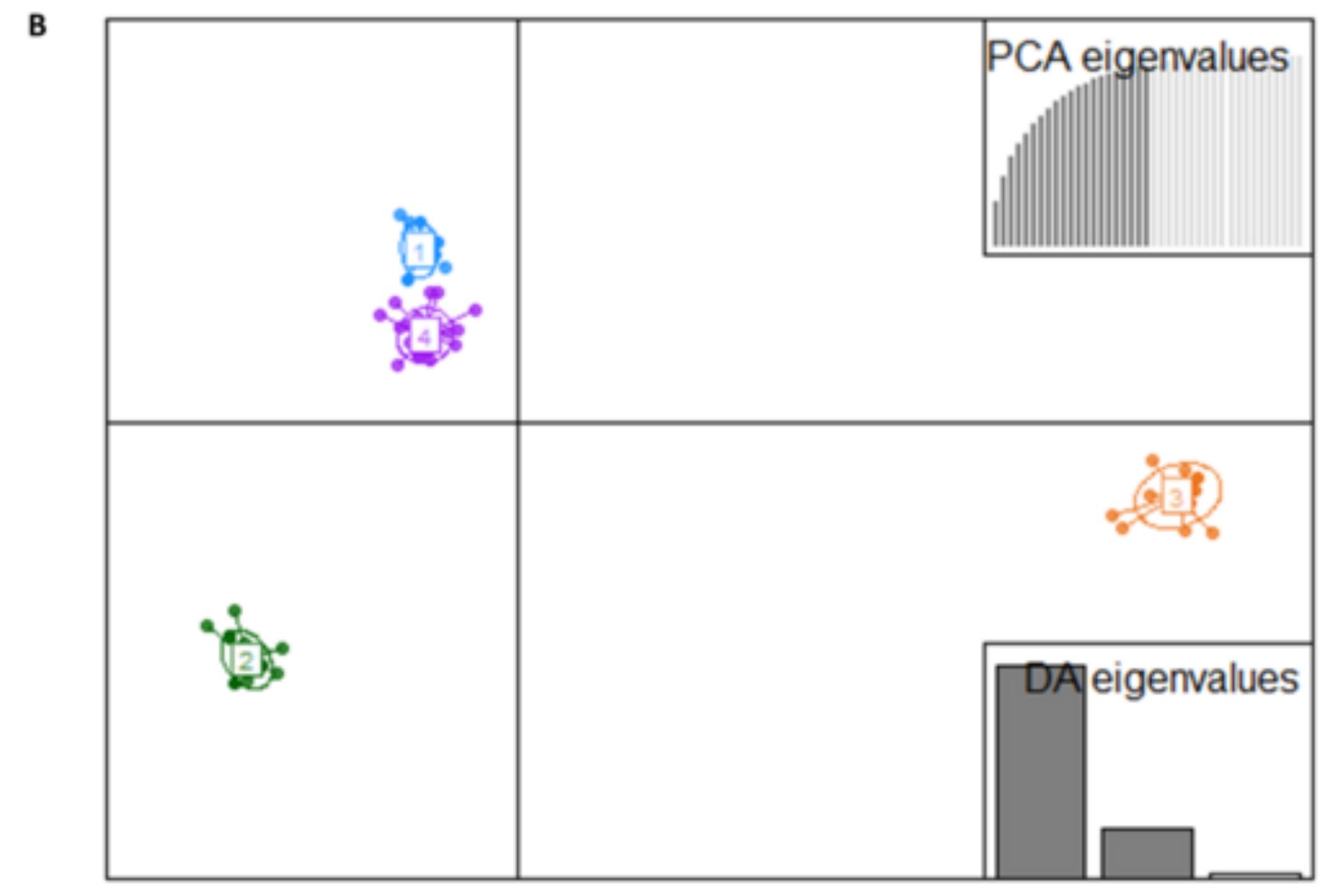

Figure 4

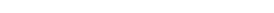

